\title{
Cuidados en América Latina: una investigación exploratoria en el contexto de la crisis sanitaria
}

\author{
Care in Latin America: an exploratory research in the context of \\ the health crisis
}

\section{Cuidados na América Latina: uma pesquisa exploratória no contexto da crise sanitária}

Romel Sergio Contreras Valenzuela*

\section{RESUMEN}

El presente texto responde a una investigación de carácter exploratorio y, como tal, estudia el caso de América Latina y cómo sus disposiciones de control para evitar la propagación del COVID-19 han tenido impacto en sus poblaciones femeninas, migrantes y personas dependientes, sobre las que se articulan las políticas del cuidado. Para ello, se hace una revisión histórica del Estado latinoamericano, donde se analizan los regímenes de bienestar que revelan la infraestructura de cuidado en la región. A continuación, se exploran las políticas implementadas en el marco de la crisis sanitaria y su transferencia de riesgo hacia las poblaciones más vulnerables al ser potenciadas por factores determinantes de la región. Por último, puesto que la pandemia es un fenómeno que se encuentra aún en progreso, se esboza un repertorio de recomendaciones y conclusiones.

\section{ABSTRACT}

This text responds to exploratory research and studies how Latin America's preventive measures to stop the spread of COVID-19 have impacted its female, migrant and dependent populations, the basis of these care policies. To this end, we make a historical
Palabras clave: COVID-19, crisis de cuidados, dependientes, Latinoamérica, migraciones, mujeres.

Keywords: COVID-19, care crisis, dependents, Latin America, 
review of the Latin American State, analyzing welfare administrations that expose the region's care infrastructure. The following section explores policies implemented during the health crisis and their transfer of risk to the most vulnerable populations as regional determining factors enhance them. Finally, since the pandemic is a phenomenon that is still in progress, a list of recommendations and conclusions is outlined below.

\section{RESUMO}

O presente texto descreve uma pesquisa de caráter exploratório e, como tal, estuda o caso da América Latina e o impacto de suas medidas de controle para evitar a propagação da covid-19 em suas populações femininas, migrantes e de pessoas dependentes, sobre as quais se articulam as políticas de cuidados. Para isso, é feita uma revisão histórica do Estado latino-americano, na qual se analisam os regimes de bem-estar que revelam a infraestrutura de cuidados na região. A seguir, são exploradas as políticas implementadas no marco da crise sanitária e sua transferência de risco para as populações mais vulneráveis ao serem potencializadas por fatores determinantes da região. Por último, uma vez que a pandemia é um fenômeno ainda em curso, uma série de recomendações e conclusões são esboçadas. migration, women.

Palavras-chave: covid-19, crise de cuidados, dependentes, América Latina, migrações, mulheres. 


\section{Introducción}

A inicios del 2020, diversos gobiernos de América Latina activaron una serie de medidas para poder enfrentar la crisis causada por la pandemia, producto de la intrusión de la COVID-19. La finalidad de estas medidas fue la de reducir la velocidad de contagio ejerciendo un mayor control de sus poblaciones. Estas disposiciones estatales consistieron en la promoción de una mayor presencia sanitaria, cierre de fronteras, confinamiento voluntario, toques de queda, restricciones en el transporte, cierre indefinido de centros educativos, etc. (Acosta, 2020). Esto produjo, como consecuencia, el debilitamiento económico de los países en cuestión, provocando una cada vez mayor tensión entre la inmovilidad y el control (Álvarez, 2020), en cuya trama se encontraron las instituciones del Estado, las familias y el mercado en una región marcada por la desigualdad.

Así mismo, el desempeño de la economía mundial ya se encontraba debilitada en el 2019, en gran parte producto de las guerras comerciales entre China y EE.UU. Sin embargo, América Latina enfrenta de forma más dramática aún la crisis sanitaria, ya que lo hace desde una posición significativamente más débil que los países del hemisferio norte (Comisión Económica para América Latina y el Caribe [Cepal], 2020b). Esto a causa de factores socioeconómicos históricos que arrastra la región como la desigualdad, la informalidad, Estados débiles, migraciones, brechas en el acceso a los sistemas de salud, escasez de políticas de cuidado, insuficientes políticas de prestación social, etc. Lo que, sumado a las pérdidas de empleo, las reducciones de las jornadas laborales y un menor porcentaje de remesas (OEA, 2020), se traduce como un mayor impacto económico.

En ese sentido, se hace necesario problematizar el hecho de que cuando hablamos del impacto económico por la crisis sanitaria, ¿̇de la economía de quién se está hablando? Ya que la crisis, tanto sanitaria como económica, y las medidas implementadas no afectan de la misma manera e intensidad a todas las personas. De hecho, se espera que el efecto de la pandemia impacte más a quienes ya se encontraban en situaciones vulnerables y con menores ingresos que el promedio. Ya que las medidas de contención de contagio significan transferencias de riesgos a quienes, además, enfrentan ahora una mayor demanda de cuidados (Lavell et al., 2020). 
En efecto, tales acciones por parte de los gobiernos no deben ser tomadas como neutras, ya que afectan más a unos grupos que a otros. Tal es el caso de las medidas de confinamiento al derivar el cuidado de grupos dependientes a mujeres y migrantes, tradicionalmente relacionados con el cuidado y la atención de las personas. De la misma forma, resalta cómo esta nueva reconfiguración del espacio devuelve a estos grupos su rol político del espacio público al espacio privado, debido al confinamiento. En ese sentido, el objetivo del presente trabajo es visibilizar y problematizar los efectos de la pandemia como de las políticas para contenerla; de qué manera se reconfiguran los cuidados en este nuevo contexto y en quiénes recaen estas labores; así como explorar sobre qué estructuras históricas y socioeconómicas acontece este fenómeno.

\section{Diseño y método}

El artículo es una investigación exploratoria acerca de la reciente problematización del cuidado en el contexto de la pandemia por COVID-19. Este consta de la revisión bibliográfica de la literatura especializada en el cuidado y en la construcción histórica de los regímenes en América Latina en materia de políticas de protección social. De la misma forma, el presente trabajo pone en diálogo los diversos aportes de la literatura en los tópicos a tratar, además de recurrir al enfoque del path-dependent que permite comprender los patrones históricos de causalidad que constriñen a eventos futuros o que, al menos, tienden a proyectarse hacia el futuro. Así, mediante el análisis de la construcción del cuidado en América Latina se propone un bosquejo de cómo la pandemia afecta los cuidados en esta región.

\section{Trabajo de campo y análisis de datos}

En primer lugar, se desarrolla la definición del cuidado y el contexto en el que surge, así como también a quiénes interpela y de qué manera. Sobre eso, se profundiza en el concepto de Estado de bienestar, así como en su genealogía en América Latina, haciendo una revisión histórica desde sus inicios y causas, con mayor énfasis en los diversos estudios acerca de las categorizaciones de los regímenes de bienestar en la región hasta la actualidad. Posteriormente, se explora la relación que se tiene con los cuidados en América Latina, las diversas presencias o 
ausencias de prestaciones sociales y los fenómenos demográficos que reconfiguran el cuidado en la región. Lo que da paso al contexto en el que se encuentra en la actualidad el cuidado y su vulnerabilidad frente a una crisis como la sanitaria y económica.

En ese sentido, la crisis sanitaria ha develado la importancia de los cuidados para el bienestar de la población frente a fenómenos como la pandemia, pero también frente a las amenazas socioculturales que ya acaecían antes en la región. Así, ante la pandemia, la evidencia de los informes frente a la COVID-19 reflejan que los países enfrentan - algunas de maneras más exitosas que otras-, las políticas de confinamiento de acuerdo con el Estado en el que se encuentren sus políticas sociales para que sus poblaciones se puedan adecuar a este nuevo contexto.

\section{Los cuidados y su ubicuidad}

Los primeros debates académicos acerca del cuidado se remontan a los años 70, mayormente en los países anglosajones, por parte de las corrientes feministas en el campo de las ciencias sociales (Batthyany, 2020). Donde se ahondó en la preocupación de visibilizar el trabajo doméstico y la conceptualización de estas tareas en relación con los procesos de producción y reproducción hasta el análisis de regímenes de bienestar alrededor del cuidado (Esquivel y Faur, 2014). Así, el cuidado se puede definir como un amplio conjunto de actividades, desde intensivas hasta extensivas, y desde empáticas y reflexivas, que al poner a disposición sus acciones pretenden resolver las necesidades de otra persona, ya que regeneran diaria y generacionalmente el bienestar físico y emocional (CEPAL, 2020b; Sojo, 2011; OEA, 2020). Por lo que se comprende que todos/as necesitamos del cuidado y/o lo necesitaremos en alguna etapa del ciclo de la vida, debido a que estos son relacionales e interdependientes (Batthyany, 2020) y engloban a los cuidados que se realizan en las personas cuidadas y que cuidan o, incluso, ejercen autocuidado (CEPAL, 2020a).

Sin embargo, tradicionalmente el cuidado se ha regido por la división sexual del trabajo, en el que se asume al hombre como proveedor natural y a la mujer como cuidadora natural. Por lo tanto, en la actualidad aún persiste esta desequilibrada distribución de las responsabilidades, donde el cuidado suele ser no remunerado (CEPAL, 2020b). Esto debido a que el trabajo no remunerado, aun siendo fundamental 
para el funcionamiento de la economía, es a menudo invisible para quienes miden la economía. Por el contrario, el cuidado minimiza el costo de reproducción de la fuerza de trabajo de quienes ejercen cuidado hacia las personas dependientes, que pueden ser niños/as, adultos mayores y enfermos, lo que lo convierte en "un factor determinante de la persistencia de las desigualdades de género en el mercado laboral" (Lamaute-Brisson, 2013, p. 23).

El cuidado, no obstante, puede ser remunerado como no remunerado. Los cuidados remunerados - que suelen ser formales o profesionales - pueden ser provistos por enfermeras/os, trabajadoras/es sociales, cuidantes, maestras/os, etc. Mientras que el cuidado informal o no remunerado puede ser provisto por familiares, amigos, conocidos, etc. Así, el informal suele ser no remunerado y no institucional, donde existen lazos de afectividad en los aspectos del trabajo pues, aunque pueden llegar a ser contradictorios, están presentes (Domínguez Alcón, 1998). De la misma forma, también se han identificado dos tendencias en los trabajos de prestaciones de cuidados. Están los que se encuentran en la esfera del hecho de criar y educar, por un lado, y aquellos cuidados que se relacionan con el trabajo de producción de la fuerza de trabajo, por el otro (Lamaute-Brisson, 2013). En la primera se desarrollan las capacidades de las personas a las que se les da el servicio y, en la segunda, se proporcionan esos cuidados para el mantenimiento de la vida y la reproducción de las siguientes generaciones.

En ese sentido, la manera en la que se aborden los cuidados posee implicancias significativas para la igualdad de género, ya que si bien puede ampliar las capacidades y opciones de hombres y mujeres, se suelen perpetuar los roles tradicionales asociados con la feminidad y la maternidad (Esquivel y Faur, 2014). De la misma forma, se atribuye a las familias el cumplimiento de este rol cuando no se tiene la posibilidad de recurrir al mercado por la prestación de servicios de cuidado. Así, en las redes intrafamiliares están presentes sentimientos de solidaridad y reciprocidad, donde además se comparten responsabilidades como también se ofrecen contraprestaciones y ayuda mutua, como parte de lo que se consideran obligaciones familiares (Domínguez Alcón, 1998). Las respuestas a estas obligaciones pueden manifestarse de formas diversas, ya sean en ejercicio y/o materiales, económicas, en 
acompañamiento y soporte emocional, recibimiento en el hogar, etc. (Calderón, 2013).

Otros aportes han buscado ir más allá de las acciones de las personas por el cuidado de personas dependientes. Esquivel y Faur (2014), por ejemplo, analizan la infraestructura para el cuidado, como el agua y la electricidad, que no suelen ser abordados como parte del cuidado (Calderón 2013). Estas demandas son relevantes, ya que después de las redes familiares, y sin acceso al mercado, solo quedan los recursos estatales. Es por ello que en la literatura la organización del cuidado es también un presupuesto de las políticas de protección social y requiere ser explorada en su dimensión política e institucional (Esquivel y Faur, 2014). Al hacerlo, se comprende que las políticas sociales responden a determinados principios y orientan la construcción de un determinado perfil de la sociedad; por lo tanto, tienen la capacidad tanto de reducir brechas de desigualdad como de perpetuarlas o, incluso, agudizarlas (Esquivel y Faur, 2014). En ese sentido, las políticas que decida implementar un Estado poseen un impacto en el bienestar de la población, como también ejercen un rol en los modelos familiares y sus dinámicas del cuidado. Este sería el caso de los países que siguieron o siguen el modelo del Estado de bienestar o, en su defecto, de regímenes de bienestar.

\section{Los regímenes de bienestar y los cuidados}

\section{Los regímenes de bienestar en Occidente}

Para Esping-Andersen (1993), el Estado de bienestar es el modelo que hace uso de acciones que implican una responsabilidad estatal que le permita asegurar unos mínimos básicos de protección social a su población. Históricamente para alcanzar el Estado de bienestar fue preciso romper con el modelo imperante de valores europeos de lógica liberal (libertad, individualismo e igualdad) en relación con individualismo, para que fuera posible la incorporación de acciones sociales por parte de un gobierno hacia los derechos de la ciudadanía (Fleury, 2021). De hecho, T. H. Marshall reconoció tres tipos de derechos: los derechos civiles o legales, los políticos o democráticos y los derechos sociales o de bienestar, donde este último, producto posterior a la Segunda Guerra Mundial, nació en forma de derecho a la seguridad social, educación, salud y protección social, entre otros (Dean, 2008). Así, 
este tercer derecho social o de bienestar supondría una igualdad de estatus en los ciudadanos de los países capitalistas.

En la misma línea, la Declaración Universal de los Derechos Humanos en 1948, y sus pactos posteriores, incorporaron la distinción que realizó T. H. Marshall y agrupó al tercer tipo de derechos como derechos de segunda generación, en los que se encontraban los derechos económicos, sociales y culturales (Dean, 2008). Asimismo, la instalación de los regímenes de bienestar europeos se sostuvo, según Esping-Andersen (1993), en dos condiciones: por un lado, la estabilidad laboral de los varones, jefes de familia; y por otro, en la estructura del empleo que se asentaba en la ya expansiva industria (Esquivel y Faur, 2014). Sin embargo, tal estructura exigía la disponibilidad de las mujeres, a tiempo completo, en su rol de cuidadoras, lo que a su vez perpetuaba el modelo de familia tradicional y heterosexual desigual.

Se ha estudiado también el Estado de bienestar desde un enfoque colectivo de movilización, en el que si bien el mercado tiende a atomizar y estratificar a los individuos obligándolos a competir - lo que limitaría su solidaridad y movilizaciones de forma colectiva- (EspingAndersen, 1993), la presencia del Estado en la desmercantilización de los cuidados por vías de políticas de protección social debilitaría tal grado de atomización. La importancia de la movilización y la posibilidad de generar esfuerzos colectivos radicaría, entonces, en la capacidad de presionar a las autoridades y aumentar así el poder de negociación. Entonces, esta capacidad de colectividad, fruto de intereses o identidad comunes, resulta ser una variable explicativa para la implementación de regímenes de bienestar, como se desarrollará más adelante.

El estudio de Esping-Andersen (1993) y su categorización en el caso europeo da luces para el inicio de la literatura especializada de Estados de bienestar. En este, el autor construye el estudio bajo el esquema de desmercantilización, desfamiliarismo y estadocentrismo-residualismo. Para ello, el primero radica en el proceso en el que bienestar se encuentra desligado del poder adquisitivo, por lo que se presta el servicio de protección social como un derecho (Esping-Andersen, 1993; Franzoni, 2005). En segundo lugar, la dinámica del desfamiliarismo consiste en poder alcanzar una vida aceptable con independencia de sus lazos fa- 
miliares. Y, por último, el estadocentrismo pone énfasis en un Estado fuerte frente a un Estado residual, que responde únicamente cuando el mercado y la familia no poseen presencia (Esping-Andersen, 1993). Bajo estos parámetros, Esping-Andersen agrupa a los países de Europa como socialdemócratas, corporativistas y liberales. Sin embargo, este modelo trae diversas críticas cuando se le quiere extrapolar al caso latinoamericano. No obstante, las dimensiones resultan útiles para comprender cómo el trabajo del cuidado recae en diferentes personas y dinámicas respecto del tipo de Estado en cuestión.

\section{El Estado de bienestar en América Latina}

América Latina fue una de las primeras regiones en desarrollar instituciones propias del Estado de bienestar después de Europa. Esto representaba, según Pierson (2005), una experiencia regional de las consecuencias del desarrollo tardío en materia de políticas sociales. Así, la experiencia latinoamericana ha sido diferente y con menor éxito que la europea, tanto en cobertura como en calidad. Además, a menudo se ha argumentado que el grupo social homogéneo en Europa habría favorecido las capacidades movilizadoras, economía cerrada y un pacto primordial por parte de sus obreros industrializados (Sojo, 2011); contrariamente a Latinoamérica, donde destaca su baja presencia industrial y alta población indígena. Sin embargo, estos rasgos generales latinoamericanos no se dan en similares proporciones en cada país, ya que América Latina es una región bastante heterogénea.

En cuanto a las políticas sociales, en el caso latinoamericano, también se ha pretendido estudiar y categorizar los regímenes de bienestar frente a las limitaciones de los estudios de Esping-Andersen (1993) para la región. Así, Mesa-Lago (2008) divide en tres grupos los Estados de América Latina. En primer lugar, se encuentran los pioneros regionales, conformado por Chile, Uruguay, Brasil, Argentina y Cuba. Estos eran países que desde inicios del siglo XX habían implementado políticas sociales como el seguro de accidentes laborales, de pensión y enfermedad-maternidad, aunque su cobertura era aún muy desigual (Del Valle, 2010; Pierson, 2005). El segundo grupo fue bautizado como los Estados intermedios y estaba conformado por Perú, México, Costa Rica, Bolivia, Ecuador, Paraguay, Colombia, Panamá y Venezuela. En este grupo el seguro social surgió después de la década de 1930 y fue 
parte de la influencia del Organización Internacional del Trabajo (OIT) y el informe Beveridge (Del Valle, 2010; Pierson, 2005). Por último, en el tercer grupo se encontraban Guatemala, El Salvador, Nicaragua, Haití, República Dominicana y Honduras. Estos países poseían una cobertura limitada e inexistente seguro laboral y asignación familiar (Del Valle, 2010; Pierson, 2005).

En línea con el modelo de Mesa-Lago, Filgueira (1998), interpretó que la división de los sistemas y sus prestaciones sociales en la región se encontraba profundamente ligada al modelo de industrialización sustitutiva de importaciones y realizó una extensión de dicho modelo, al estar influenciado en Esping-Andersen (Del Valle, 2010; Franzoni, 2008). Así, para el autor, en el primer tipo de régimen, llamado universalismo estratificado, se encontraban Uruguay, Argentina y Chile. Este se situaba cercano al modelo conservador de Esping-Andersen, ya que poseían políticas extendidas, pero segmentadas por ocupaciones, y coincidían en el temprano desarrollo de sus sistemas de bienestar (Del Valle, 2010; Franzoni, 2008). En el segundo grupo se encontraban los regímenes duales, esto es, Brasil y México, donde la estratificación era más acentuada que en el grupo anterior (Del Valle, 2010), aunque excluía a poblaciones en las zonas rurales (Franzoni, 2008). En tercer lugar, estaban los regímenes excluyentes, compuestos por El Salvador, Guatemala, Honduras, Ecuador, República Dominicana, Bolivia y Nicaragua. Este último grupo constaba de un sistema elitista y excluyente, con una distribución de recursos casi inexistente (Del Valle, 2010; Franzoni, 2008).

Por su parte, el estudio de Franzoni rescata las dimensiones mencionadas de desmercantilización, desfamiliarización, pero añade el desempeño (Pribble, 2011), donde además aborda nociones como el cuidado y el rol de género en las políticas sociales. Así, identifica a tres grupos. El primero lo cataloga como estatal de proveedor único, en el que se encuentran Costa Rica y Uruguay. Estos países, según Franzoni (2005), son excepciones en cuanto a las condiciones de vida y, además, atendiendo al índice de Gini, poseen menor desigualdad. En el segundo grupo, la autora toma como ejemplo a México, Argentina y Chile (Franzoni, 2005) y los cataloga como régimen de bienestar liberal único. En esta categoría la inversión pública sigue siendo alta y combina asignaciones completas con asignaciones focalizadas. En último lugar, 
el régimen de bienestar informal de doble proveedor, donde se encuentran El Salvador, Guatemala y Nicaragua. En este grupo, el coeficiente de Gini es mayor a 60, las familias cuentan con doble proveedor, la informalidad es alta y existe escasa presencia y capacidad institucional (Franzoni, 2005).

Otro modelo que busca categorizar a la región es el de Jennifer Pribble (2011), quien presta mayor atención a los determinantes de los anteriores modelos, por lo que además de recurrir a los factores estructurales, pone énfasis en los de carácter político. En su modelo integra y realza el factor de la industrialización, de los movimientos colectivos, de la población étnica e indígena, etc. Así mismo, define a los regímenes de política social como "el paquete de políticas proporcionadas por el Estado que protegen a los ciudadanos de la pobreza y redistribuyen los ingresos y las oportunidades de vida" (Pribble, 2011, p. 192). En la misma línea, la autora, para describir la división de los países de la región, elabora dos dimensiones de políticas: Prevención de riesgos (risk prevention) y Afrontación de riesgos (risk coping). En el primero se encuentran las políticas encargadas de mejorar los niveles de vida y el capital humano como forma de evitar la pobreza. El segundo radica en "el intercambio directo de beneficios en efectivo y, por lo tanto, proporcionan a los políticos recursos que son útiles para construir vínculos corporativos" (Pribble, 2011, p. 194).

En este modelo, construido por Pribble (2011), en el grupo uno se encuentran Chile, Costa Rica, Uruguay y Argentina, caracterizándose por un buen desempeño tanto de prevención de riesgos como de confrontación de riesgos. Además, constan de experiencia en industrialización, salvo Costa Rica, además de mayor fortaleza democrática y homogeneidad étnica. En el segundo grupo de políticas de protección están Panamá, México y Brasil, con alta calidad de afrontamiento de riesgos, pero no así en prevención de riesgos. Este grupo posee reducido nivel de movilización del sector popular y presencia de líderes autoritarios, haciendo posible la generación de políticas de arribaabajo (Pribble, 2011). La tercera configuración de protección social está compuesta por Colombia, Ecuador Paraguay y Perú, en la que hay niveles intermedios de afrontamiento de riesgos como de prevención de estos. En este grupo estuvo ausente la incorporación política tanto por movilizaciones como por organizaciones corporativas. Asimismo, 
existen altos niveles de poblaciones étnicas e indígenas, lo que produciría mayor heterogeneidad y dificultad para generar unidad, lo que se traduciría como menos incentivos para que los líderes concedan políticas que beneficien a estas poblaciones. Por último, el cuarto grupo está ejemplificado por Nicaragua, Guatemala, El salvador y República Dominicana. Este grupo se caracteriza por su reducida capacidad de confrontación y prevención de riesgos. En este grupo, según la autora (Pribble, 2011), la industrialización estuvo ausente y persistió la presencia de autoritarismos arraigados a la agricultura que no generaron políticas de protección social.

Para efectos de este artículo, revisar los distintos trabajos que generaron clusters de los regímenes de bienestar de la región es fundamental, debido a que permiten identificar las variables que han logrado cambiar o perpetuar la disposición de los distintos tipos de Estados al brindar políticas sociales. De la misma forma, los esfuerzos por introducir en la literatura estos estudios desde diversos enfoques y preocupaciones enriquece la comprensión de los Estados en la región. Evidencian, además, que desde el siglo pasado, tras varios cambios económicos y políticos, los países tienden a concentrarse en los mismos grupos. No existen grandes alteraciones, pero sí mayores precisiones y nuevos enfoques respecto de las causas de sus posiciones. En ese sentido, la construcción de los Estados capaces de brindar políticas de bienestar a sus poblaciones responden a patrones históricos, pero también a la proyección de estos, es decir, patrones ya sean construidos o heredados. Así mismo, llama la atención cómo los autores ponderan los altos niveles de heterogeneidad que existe en América Latina, lo que dificulta poseer un solo análisis determinante, pero que también resalta aquellos que tienen en común la desigualdad, informalidad, dependencia económica, etc. (Esquivel y Faur, 2014; Franzoni, 2005; Mesa-Lago, 2008; Pribble, 2011).

Así, en el siglo XX en América Latina, gran parte de la población no contó con acceso a servicios de salud, ingresos estables y pensiones. De hecho, aun que cuando existió protección social para los trabajadores, esto solo se implementó en el sector formal, más no en el amplio sector informal (Garay, 2016). Por lo tanto, el sector formal fue el común protagonista de los beneficios sociales en la región, debido a la corriente de industrialización, también llamado desarrollismo; no 
obstante, su adquisición no fue inmediata. De hecho, la consigna de conseguir para América Latina más derechos -incluso para el sector formal-de carácter social ha sido heterogénea. En algunos casos fue, como se comentó líneas arriba, por fuerza de las luchas y colectivos sociales, pero en otros, surgió por parte de líderes autoritarios. Huber (2009), por su parte, intenta explicar el por qué de la adquisición de estos derechos en la región. En su estudio encuentra una alta correlación en la que los largos períodos de democracia hacen posible que emerjan organizaciones que representan intereses en común, mientras añade que los partidos políticos de izquierda direccionen la mirada estatal hacia un sentido más progresista, lo que significa una mayor asignación en el gasto público para afectar la distribución, como es el caso de las leyes de salario mínimo.

En cuanto al sector informal en la región, si bien es cierto que existía mucho antes de la década de los 80 , es recién en esta época cuando suscita el interés de los académicos. Hasta entonces, América Latina había logrado implementar periodos de industrialización en la mayoría de sus países, algunos más exitosos que otros, pero cuyos beneficios solo se extendían al sector industrial y formal. En efecto, existe evidencia de que estos períodos de industrialización dieron lugar a lo que fue bautizado como Estados de bienestar truncos, caracterizados por proporcionar beneficios de seguro social a un grupo reducido de trabajadores formales (Holland y Schneider, 2017). Mientras que el sector informal se encontraba en relaciones clientelares con los líderes de turno y fragmentados por su condición heterogénea. Esta distribución laboral se mantuvo sin muchos cambios hasta la "crisis de la deuda" a inicios de la década de los 80 . A lo que los países de la región respondieron con la implementación de reformas orientadas hacia una severa contracción estatal, seguida de una marcada privatización de prestaciones y empresas que hasta el momento eran nacionales (Garay, 2016), lo que empujó a gran parte de la población formal al sector informal.

Así, estos ajustes estructurales en los 80 , agudizados en los años 90, fueron implementados en el marco del Consenso de Washington, además de los organismos internacionales que dictaban una serie de recetas de corte neoliberal para la región. Estas reformas significaron también la liberalización del sector salud, la mercantilización y re- 
tracción de los beneficios antes brindados por el Estado. Por lo que, posteriormente, dio paso a un largo proceso de mayor segmentación $\mathrm{y}$ fragmentación que generó profundas desigualdades en el acceso a prestaciones sociales como pensiones y salud de calidad (Bohoslavsky y Rulli, 2020). Así mismo, estas reformas, como la desindustrialización, aumentaron "la importancia numérica de los trabajadores que corren el riesgo de caer en el sector informal" (Holland y Schneider, 2017). De hecho, estas reformas también incrementaron la pobreza en la región, además de que - como argumentan los especialistas-, la inequidad ralentiza el crecimiento (Franzoni, 2005). Por lo tanto, es en este contexto donde la informalidad y la precarización se incrementan robustamente en América Latina.

Este proceso de informalización en la región ha sido ampliamente estudiado, debido a que se le ha considerado como un obstáculo para el desarrollo económico y el bienestar social. Se sabe, pues, que la población informal tiene menos acceso a beneficios sociales, además de encontrar barreras colectivas, escasos recursos y débil infraestructura organizativa para hacer frente a las políticas que no les excluya (Garay, 2016). En ese sentido, entre los estudios acerca de la informalidad en la región destaca el de Guillermo Perry et al. (2007), quienes definen la informalidad como la interacción imperfecta entre los ciudadanos y su Estado, y encuentran que las grandes empresas son menos propensas a la informalidad, debido a su organización, siendo desproporcionadamente mayor el caso de pequeñas empresas en América Latina (Camargo y Caicedo, 2012). Asimismo, identifica dos razones de ser de la informalidad en la región: por exclusión y por escape (Perry et al., 2007). El caso de la exclusión, la informalidad radica en las barreras que impone el Estado a las empresas y que impide que estas alcancen la frontera de la formalidad, perjudicando también a sus trabajadores. En el caso del escape, surge la evaluación costo-beneficio de los empresarios respecto de las poco atractivas prestaciones que otorga el Estado, por lo que se tiende a elegir la informalidad.

Por lo tanto, se suelen cuestionar las medidas del paquete de neoliberalización en América Latina. De hecho, se argumenta que el sistema tributario de carácter ortodoxo que se implementó fue un fracaso, ya que se había pensado en un modelo europeo y no local. Es decir, 
se ignoró — según Del Valle (2010)—, una serie de rasgos como el alto nivel de evasión, debido a la magnitud informal; el bajo nivel de moral fiscal, maniobras fraudulentas, etc.; y la limitada capacidad de control de las entidades tributarias y su elevado gasto fiscal. Lo que devino en una región que, si bien antes priorizaba la dualización en su bienestar, ahora tendía incluso a mayor fragmentación de sus relaciones sociales (Del Valle, 2010). Por otro lado, este periodo de neoliberalización estuvo también caracterizado por un mayor aumento de la acumulación del capital, ausencia de pensiones de asistencia social, mayor desigualdad de género, fallas de integración, altos costos fiscales de transición, etc. (Mesa-Lago, 2008). De esta forma y sin mayores cambios, bajo la lógica neoliberal América Latina atraviesa el siglo XXI con altos niveles de desigualdad e incertidumbre económica.

\section{Los cuidados en América Latina}

Producto de los cambios en políticas económicas de finales del siglo XX, América Latina empieza el siglo XXI con serios problemas de distribución y eficacia en cuanto a protección social. En efecto, la distribución de los cuidados se encuentra altamente desequilibrada, derivándolas a los hogares y siendo ejercidas mayoritariamente por mujeres de forma no remunerada (ONU-Mujeres y CEPAL, 2020). De hecho, en el mundo, las tres cuartas partes del cuidado no remunerado lo realizan las mujeres y en América Latina, específicamente, el $75 \%$ de ellas realizan el trabajo doméstico y de cuidados no remunerados (Bohoslavsky y Rulli, 2020). Además, el trabajo de cuidados ha sido históricamente invisibilizado e, incluso, desprestigiado. Así mismo, esta desprotección de las mujeres, sumada a la sobrerrepresentación de ellas en el trabajo informal y sus bajos ingresos, las expone a condiciones adversas en su cotidianeidad (ONU-Mujeres y CEPAL, 2020).

Por lo tanto, se evidencia que la organización social de cuidados ha sido elaborada y legitimada bajo una estructura desigual fundamentándose, como se ha escrito al inicio, en una división sexual del trabajo mediante roles estereotipados. No obstante, en las últimas décadas, el ejercicio de los cuidados se problematiza más a medida que surgen cambios sociales, culturales, y demográficos como el inevitable “progresivo envejecimiento de la población, la diversificación de los patrones familiares, el ingreso masivo de las mujeres al mercado labo- 
ral y en otras esferas de la vida social" (ONU-Mujeres y CEPAL, 2020), los que generan una crisis mayor aun en la región que, a su vez, afecta la demanda y oferta de los cuidados. Esto significa que América Latina inicia este siglo afrontando nuevos desafíos en materia de políticas públicas bajo un modelo neoliberal aún muy presente en varios países de la región.

Por un lado, la crisis de los cuidados respecto de su capacidad para garantizarlos se debe a diversos motivos. Entre ellos, se encuentra la transformación de la estructura familiar que ha ocurrido en las últimas décadas. Destacan así la reducción familiar y la creciente participación laboral femenina (Aranco et al., 2018). De hecho, la participación laboral de mujeres en América Latina y el Caribe pasó de 20\% en 1960 a 50\% en el 2015 (Aranco et al., 2018). Sin embargo, este incremento se debe, en gran medida, a la aspiración por una identidad propia, autonomía e independencia económica; significa también, en los espacios menos privilegiados, una doble carga para las mujeres que no cuenten con la disponibilidad de cuidados, ya sea mediante servicios públicos o servicios mercantilizados (Calderón, 2013). Recordemos que lo que hará que el cuidado dependa más del mercado, del Estado o, a falta de ambos, de la familia dependerá del tipo de regímenes de bienestar implantado en un Estado.

Por otro lado, aunque es pronto aún para considerar a la población de América Latina como envejecida, la tendencia en los próximos años parece indicar que ese es el camino, como lo es también en regiones como Europa. Sin embargo, el ritmo actual de envejecimiento no tiene precedentes en la región. La población mayor de 60 representa el $17 \%$ de la población y se estima que para el 2050 llegue al 25\% de la población (Aranco et al., 2018). Lo que se traduce, en las condiciones actuales, como una mayor presión sobre los responsables del cuidado, en su mayoría, mujeres, debido a la mayor demanda de cuidados por parte de la población adulta, ya que aumenta las limitaciones funcionales que potencian las causas de discapacidades (Aranco et al., 2018; Rossel, 2016). Asimismo, se espera que exista una mayor presión por sistemas de jubilaciones y pensiones y de mayor atención en el sector salud (Rossel, 2016). Este proceso de envejecimiento refuerza la crisis de cuidados y limita algunas potencialidades de América Latina que derivaban del bono demográfico 
que se encontraría aproximando su fin, además del elevado descenso de la fecundidad actual.

La migración, a su vez, también es un fenómeno que afecta los cuidados y su crisis. Tradicionalmente, las migraciones se han realizado de América Latina hacia los países más desarrollados en Occidente o América del Norte. Esta población migrante — que tiende a ser más joven y a tener mayores tasas de fertilidad-, ha tendido a contrarrestar, en parte y no de forma suficiente, el proceso de envejecimiento en aquellos países de su destino (Rossel, 2016), pero generando el sentido contrario a sus países de origen. Lo que además ha sido catalogado por Bettio et al. (2006) como "fuga de cuidados". Sin embargo, debido a las medidas restrictivas y antimigrantes por parte de los países más desarrollados, las olas migratorias suelen ser cada vez más regionales. En tal sentido, las poblaciones migratorias carecen de acceso a políticas sociales en países con ya escasos sistemas de bienestar, donde se les asigna una posición más vulnerable que la de solo informal al ser además extranjeros. Es ahí donde también suelen aportar en la organización social de cuidados como trabajadoras/es del servicio doméstico y que, como explican Esquivel y Faur (2014), son cada vez más las mujeres migrantes quienes ocupan este rol al servicio de las clases medias urbanas de los países de destino. Este proceso en el que se delega a otras mujeres la división social del cuidado es también conocido como tercerización de los cuidados.

Así, en este mundo globalizado en el que nos encontramos, sujeto a los sistemas económicos y sociales imperantes, este proceso de migración por parte de mujeres en el cuidado ha originado lo que ha sido llamado cadenas globales de cuidado. En este proceso migratorio se transfiere el cuidado desde los hogares de los países de origen hacia aquellos de los países receptores, surgiendo familias transnacionales donde la maternidad, por ejemplo, se realiza a distancia (Sojo, 2011). Esta división internacional del trabajo o transferencia internacional del cuidado se caracteriza porque a medida que descienda más la cadena, así también lo hace el valor asociado al cuidado, donde los migrantes suelen tener más dependientes a su cargo y, además, en sus países de origen en los que habrá, también, otras mujeres implicadas en el cuidado de forma no remunerada (Rossel, 2016). Asimismo, las migraciones, en ese contexto, suelen contribuir económicamente como sostén 
de las familias y, por lo tanto, de los cuidados por medio de remesas de un país a otro.

Como ya se ha mencionado antes, la informalidad y la desigualdad son fenómenos con mucho protagonismo en la región. No sorprende, pues, que se encuentren vinculados, por lo que revisarlos ayuda a comprender mejor a los Estados latinoamericanos en la actualidad y su crisis de cuidados. Producto de las reformas de desindustrialización y liberación de los mercados, muchos trabajadores y las familias más pobres fueron excluidos del sistema. En ese sentido, estos/as trabajadores/as informales suelen tener resistencia al acatamiento de las leyes impositivas, acción que depende inversamente de las "percepciones de 'captura del Estado' y positivamente de las percepciones de la calidad del gasto público" (Perry et al., 2007). Por lo tanto, se deduce una baja confianza hacia el Estado y, por ello, existe poca responsabilidad tributaria y cumplimiento de las normas (Camargo y Caicedo, 2012). De ahí que la desigualdad entre ingresos y poder esté correlacionada con la informalidad y asociada también con instituciones débiles y posibles capturas del Estado (Perry et al., 2007). Esta es la realidad de muchos países de América Latina, donde el Estado es incapaz de hacer cumplir las normas y, además, es visto como insuficiente proveedor de bienes meritorios, lo cual "se refleja en la desigualdad de oportunidades y mala distribución del ingreso" (Camargo y Caicedo, 2012).

\section{Crisis sanitaria en los cuidados en América Latina}

En la actualidad, debido a la ausencia de grandes cambios en las últimas décadas, la mayoría de países latinoamericanos posee aún Estados pequeños con bajos ingresos tributarios y bajos gastos públicos, donde además los recursos que se utilizan no se encuentran bien designados (Huber, 2009). La calidad de las democracias, por su parte, sigue siendo defectuosa en su mayoría con serios problemas políticos. En ese sentido, es aún vigente la expresión de Centeno y Ferraro (2013) acerca de Latinoamérica como una región de "leviatanes de papel" por sus Estados débiles y su poca legitimidad, poca capacidad para hacer efectivas sus ordenanzas de leyes, baja carga impositiva, dificultad para sostener políticas públicas en general, etc. Asimismo, en la región, los servicios sociales aun cuando aumentan, lo hacen de manera desigual en calidad y acceso para los grupos más pobres, y las principales trans- 
ferencias que se efectúan por programas de seguridad social tienden a ser cada vez más regresivos (Huber, 2009).

Asimismo, el trabajo no remunerado continúa siendo casi exclusivamente ejercido por parte de mujeres y los estudios acerca del uso del tiempo no muestran modificaciones sustantivas en el reparto de tareas domésticas (Franzoni, 2005). Si bien la inserción laboral femenina es un fenómeno en constante crecimiento, la diferencia entre hombres y mujeres sigue siendo alta: casi la mitad de las mujeres en la región se encuentra trabajando, frente a las tres cuartas partes en el caso de los hombres; además, el trabajo a tiempo parcial es practicado por una de cada cuatro mujeres, mientras que en los hombres es uno de cada ocho (Gutiérrez et al., 2020).

Como se aprecia, ya antes incluso de la pandemia, América Latina era una región muy desigual para las mujeres, pero también para otros grupos, como lo demuestran Gutiérrez et al. (2020) en un estudio con enfoque interseccional: los mercados de trabajo se encuentran amenazados y sin mayor respaldo sobre las mujeres de bajos ingresos, habitantes en zonas rurales, jefas/es de hogar y jóvenes (15 a 24 años de edad). Así mismo, América Latina también ha seguido sufriendo los estragos de la crisis financiera del 2008. La tasa de crecimiento del PIB regional se redujo de un $6 \%$ a 0,2\% e, incluso, en el periodo de 2014-2019, el crecimiento fue el menor desde 1950: 0,4\% (CEPAL, 2020a). Además, los esfuerzos por reducir la desigualdad han sido insuficientes, pues el espacio fiscal ha sido bastante limitado, como lo han sido también los ingresos públicos. La crisis financiera y su ralentización en la actividad económica, la evasión fiscal y el fin del alza de las materias primas ocasionó un estancamiento en la región. De esta forma, en la última década, los ingresos públicos totales por parte de los gobiernos centrales latinoamericanos promediaron 18,2\% del PIB (CEPAL, 2020a).

De la misma forma, los efectos desde el periodo de neoliberalización sobre el sistema de salud han tenido un alto y largo impacto. La cobertura regional desde 1990 a 2001 se redujo en sus formas estatales, lo que ocasionó que se aumentara en sus versiones privadas, aunque estas con menor cobertura que aquellas, provocando, así, menor acceso a las poblaciones vulnerables. Esto ocasionó un mayor predominio de mecanismos contrarios a la solidaridad y agravó la desigualdad de género debido a la "selección de riesgos practicada contra las muje- 
res en edad fértil" (Mesa-Lago, 2008). En ese sentido, en la actualidad, frente a la COVID-19, América Latina se encuentra en una posición más vulnerable que otras regiones del mundo, ya que cuenta con sistemas de salud caracterizados por ser débiles y fragmentados, que por lo tanto no garantizan un acceso universal necesario frente a una crisis como la sanitaria ocasionada por la pandemia (CEPAL, 2020b). Además, históricamente los sistemas de salud se han situado en espacios geográficamente centralizados, lo que repercute en un todavía menor acceso para las poblaciones rurales o periférico-urbanas.

Así, la magnitud de la estrechez fiscal y la debilidad de los Estados, propias de la región han comprometido a su vez otros cuatro sistemas de protección social que se ven afectados para hacer frente a la pandemia (CEPAL, 2020a). Por un lado, el aumento de la informalidad, lo que ocasiona mayores brechas en acceso de protección social y mayor aumento de trabajo por cuenta propia. En segundo lugar, existen muy pocos países en la región que cuenten con prestaciones de desempleo. En tercer término, inevitablemente, los sistemas de protección social de carácter contributivo se verán afectados por una cada vez mayor demanda de prestaciones de licencias por enfermedad, al menos en el sector formal. Por último, la reducida cantidad de programas de protección social de orden no contributivo repercutirá en el ya poco apoyo a los pobres y a grupos de bajos ingresos.

Sumado a esto, un estudio de CEPAL (2020a), prevé que las familias con menores ingresos se verán obligadas a mandar a sus hijos/as al trabajo, lo que significará una mayor tasa de trabajo infantil en la región. De igual modo, otros estudios estiman que las empresas más grandes y con mayores recursos podrán hacer frente a la crisis económica producto de la crisis sanitaria, mientras que eso no será así para las empresas pequeñas, las que aunque pueden recuperarse más rápido (Lavell et al., 2020) tienen incertidumbre al respecto, ya que depende del tiempo de paralización o ralentización de la economía. Lamentablemente, en América Latina, casi el 99\% de las empresas son micro, pequeñas o medianas y, además, constituyen la mayor parte en casi todas las áreas de economía regional (CEPAL, 2020b).

De esta manera, la crisis sanitaria afecta económicamente a toda la región, pero golpea aún más a poblaciones que ya se encontraban vulnerables en el día a día. De hecho, a diferencia de otras crisis, esta 
sucede en un periodo de mayor desigualdad en el orbe. Producto de las medidas de confinamiento que exigen controlar el movimiento mediante el cierre de fronteras y la inmovilización masiva, se ha visto afectada negativamente la población migrante. Así, en un periodo sin precedentes, el movimiento global de refugiados, migrantes por anteriores crisis nacionales, desplazados, etc., se ve condicionado por la crisis sanitaria. Esto en el marco de un proceso, no de internacionalismo, sino de hipernacionalismo, que coloca a los migrantes en una posición de no-nacional, frente a los ciudadanos nacionales, donde aquellos son irregularizados, criminalizados, se tornan en cuerpos parecen no merecer la defensa, sino más bien la exposición al contagio y la muerte durante la pandemia (Álvarez, 2020; Buttler, 2020).

Por lo tanto, la crisis sanitaria devela la ya injusta organización social/sexual del trabajo. Pues, datos como la sobrerrepresentación de las mujeres en el trabajo del sector informal o en aquellos de menores ingresos "dejan expuestas las condiciones adversas de la crisis y refuerzan los vínculos perversos de la pobreza monetaria y la pobreza de tiempo" (ONU-Mujeres y CEPAL, 2020). En efecto, incluso antes de la COVID-19, las mujeres en la región destinaban entre 22 y 42 horas semanales a las actividades domésticas y de cuidados (CEPAL, 2020c). De hecho, estas desigualdades, incluso, se incrementan en los hogares de menores ingresos, donde la demanda de cuidados ya era aún mayor y ahora se agudiza más, debido a que la atención de salud se traslada a hogares, sin políticas de corresponsabilidad (CEPAL, 2020c).

Si bien la COVID-19 ha desatado una crisis sanitaria y económica, ha exigido por parte de los Estados una respuesta inmediata frente a estas nuevas adversidades. En ese sentido, las políticas públicas desplegadas por parte de los gobiernos latinoamericanos no han sido, en su mayoría, elaboradas desde un enfoque de los cuidados. De hecho, desde una perspectiva de gestión de riesgos, se puede afirmar que muchas de las medidas implementadas transfirieron riesgos hacia las poblaciones menos privilegiadas. En este tema, Lavell et al. (2020) sostienen que existieron tres categorías de riesgos mutuamente relacionados. En primer lugar, el riesgo primario de la materialización del virus y su mortalidad. En segundo término, el estrés o colapso de los sistemas sanitarios y de las morgues o servicios funerarios. Por último, las políticas implementadas para evitar el desborde del primer y segundo 
riesgo, lo que se tradujo en medidas de confinamiento o paralización económica.

De esta manera, las consecuencias, producto de intentar evitar los riesgos primarios y secundarios, a menudo, recaen en grupos específicos. En este caso, los riesgos económicos y sociales asociados con las medidas que buscan evitar la propagación del virus serán mayores para sectores sociales como la población migrante, trabajadores/as informales, trabajadoras sexuales, personas sin hogar, mujeres en trabajos domésticos remunerados o no remunerados, etc. Para estos sectores, argumentan Lavell et al. (2020), es posible que la lucha diaria por la supervivencia, la exposición y su repetida experiencia en salud de baja calidad, desnutrición crónica, delincuencia y otros riesgos asociados que genera el encierro para reducir las probabilidades de infección por el virus SARS-coV-2, terminen siendo un riesgo mayor que ignorar las recomendaciones de confinamiento. Esto debido a que la situación les obliga a salir a buscar un ingreso y sustento diario. Por ello, sugieren los autores, es menester que las políticas públicas deban enfocarse en si las externalidades transferirán riesgos a poblaciones que ya se encontraban vulnerables.

No muy diferente de las formas en las que estas políticas se realizaron, han sido aquellas recomendadas históricamente por las Instituciones Financieras Internacionales (IFI). El despliegue de estas instituciones frente a la crisis sanitaria fue casi inmediato. Entre las que destacan por las grandes cantidades de dinero que son capaces de ofrecer en sus ofertas de crédito se encuentran el Banco Mundial (BM) y el Fondo Monetario Internacional (FMI). Sin embargo, estas instituciones suelen imponer condiciones al momento de entregar un préstamo. Así, en el pasado, y con más fuerza desde los 80 fruto de las recetas de estas instituciones, surgieron los ajustes estructurales caracterizados por políticas ortodoxas de alta austeridad. Lo que devino en Estados reducidos con poca capacidad y alcance estatal, así como un adelgazamiento en las políticas de protección social, al privatizar la salud o desaparecer los servicios. No obstante, Bohoslavsky y Rulli (2020), evidencian que estas medidas no solo impactaron de forma desproporcionadamente negativa a las poblaciones pobres y agravaron la desigualdad en la región, sino que también afectaron más a las mujeres. 
Por ello, en su trabajo Bohoslavsky y Rulli (2020) argumentan que las medidas, históricamente producidas por estas instituciones, han resultado ser androcéntricas, ya que socavan la igualdad de género y los derechos humanos de la población femenina al privatizar los servicios públicos, pues entre sus lineamientos está

reducir el tamaño de las redes de seguridad social y recortar los salarios, junto con la desregulación laboral, las reducciones en las pensiones, y la profundización de regímenes tributarios regresivos mediante la introducción o el aumento del IVA, al tiempo que se reducen las tasas impositivas empresariales. (Bohoslavsky y Rulli, 2020, p. 5)

No obstante, ahora frente a la crisis sanitaria, una vez más estas instituciones vuelven a ofrecer medidas similares de austeridad en la lógica neoliberal. Por ello, sostienen los autores, tales recomendaciones y condicionalidades no han sido diseñadas con un enfoque de género que reconozca la injusta organización social del cuidado que padece esta región.

\section{Resultados}

Se puede deducir que al recaer el mayor impacto económico sobre estos grupos vulnerables, se agudiza todavía más la crisis de los cuidados, pero además amplía las brechas entre ricos y pobres. En materia de los cuidados, las personas que se encontraban excluidas del sector formal lo estaban también de las prestaciones sociales, mientras que los que pertenecían a los sectores formales se encontraban favorecidos, lo que ya aumentaba la brecha (Pribble, 2011). No obstante, es ahora que estas brechas se distancian aún más, ya que los que poseen menos recursos se ven empujados al sector informal y, por lo tanto, tampoco reciben apoyos por parte del Estado que les permita hacer frente a la crisis sanitaria y económica, por lo que las demandas de cuidados no encuentran amparo en el Estado ni tampoco en el mercado, relegando a las familias la gestión de estas tareas.

Así mismo, es importante recalcar que estas diferencias en el impacto de la COVID-19 no son las mismas incluso entre países de América Latina. En efecto, e países con mayor formalidad como Chile y Uruguay, mientras que hay otros que por lo contrario muestran una alta 
tasa de informalidad que se viene en aumento por la crisis sanitaria, como es el caso de Perú y Bolivia. Un ejemplo de ello son Bolivia, Perú y Guatemala, donde más del $80 \%$ de mujeres se encuentra en el sector informal (Bohoslavsky y Rulli, 2020). Nos encontramos, pues, con una región que - como se ha demostrado en las categorizaciones de los regímenes de bienestar- es altamente heterogénea intrarregional e intranacionalmente, tanto desde la forma en la que se han construido sus políticas sociales como en cuanto a los insumos para la satisfacción de necesidades. Los patrones de respuesta frente al COVID-19, por su parte, parecen mantenerse, lo que lleva a que los países con mayor protección social tendrán mejores sistemas de salud y más homogeneidad para responder mejor a la epidemia. Así, la evidencia sugiere que aquellas naciones que no gocen de beneficios sociales sufrirán un mayor impacto económico, pero también en su salud. De hecho, la tasa cruda de mortalidad por COVID-19 (TCM) se encuentra asociada a personas que viven con menos de un dólar al día (Acosta, 2020).

Por otro lado, en la línea de políticas para responder la pandemia, las medidas de confinamiento recaen también en las poblaciones más vulnerables. De la misma forma, la crisis económica y las políticas que buscan reducir su impacto lo hacen con las poblaciones menos privilegiadas. De hecho, no es la primera vez que las medidas de ajustes y austeridad han dejado a América Latina en una posición vulnerable frente a cualquier fenómeno como lo es ahora el de la crisis sanitaria por la COVID-19. Entonces, si es que las políticas de ajuste y de minimización del Estado - y por lo tanto de sus prestaciones sociales- generan mayor número de personas en el sector informal, habrá menos acceso a la salud pública y a los beneficios sociales, de modo que los costos para la organización social del cuidado también serán mayores. Es preciso mencionar que la pobreza viene acompañada por muchos factores que complican el aislamiento social que requiere el tratamiento de este coronavirus, entre ellos: la falta de viviendas, el hacinamiento, menores accesos a los servicios de salud, alimentación insuficiente o de mala calidad, falta de agua potable y obstáculos económicos para teletrabajar o acceder a la educación a distancia.

Si bien, como ya se señaló, los países latinoamericanos y sus sistemas de políticas sociales - y por lo tanto sus cuidados- ya se encontraban comprometidos antes de la crisis sanitaria, este nuevo fenó- 
meno significa una amenaza para las ya debilitadas instituciones del cuidado. Por su parte, las respuestas y evoluciones de la pandemia por la COVID-19 parecen estar en línea con sus heterogéneos regímenes sociales. Esta heterogeneidad se asociaría tanto con las particularidades de cada país como con el tamaño de su población, los niveles de pobreza urbana, las medidas de salud adoptadas y las condiciones sanitarias preexistentes (Acosta, 2020). De igual modo, debido al confinamiento como respuesta estatal ante la crisis sanitaria más niños/ as en edad escolar, adultos mayores dependientes vulnerables, personas con discapacidad o movilidad reducida deben permanecer en el hogar, lo que se traduciría como un mayor estrés para las personas que asumen los cuidados, siendo en su mayoría en la región, mujeres y migrantes. Eso deja en evidencia que la crisis sanitaria y sus externalidades poseen un mayor impacto en las personas sobre las que se articulan los cuidados, es decir, en los/as cuidadores y en los/as cuidados.

\section{Discusión y conclusiones}

Al ser un fenómeno todavía en proceso, la crisis sanitaria y económica y sus efectos en los cuidados evidencia una serie de limitaciones para la investigación. En ese sentido, por la naturaleza de la investigación, ha sido posible explorar la problematización del cuidado y dar luces acerca de las causas del efecto en el objeto de investigación, haciendo uso de diversas fuentes, más no es posible tener completa certeza acerca de los efectos de la causa, ya que la pandemia todavía no ha terminado y los informes al respecto son solo fotografías del momento. Sin embargo, el trabajo pretende al menos dar un esbozo en la región de lo que sería la película de algunos grupos menos privilegiados en relación con el cuidado frente a la crisis sanitaria.

Por ello, al concluir este trabajo se otorga la licencia de brindar recomendaciones respecto de cómo optimizar los cuidados en América Latina. Por lo tanto, las políticas públicas que no aborden las complejidades de la región se verán obstaculizadas al momento de implementarse. Reconocer las brechas de igualdad entre género, pero también entre sectores sociales será primordial en ese sentido, como también reconocer la heterogeneidad de la región. De la misma forma, se deben buscar políticas que permitan a las personas quedarse en casa sin pasar hambre, sin amenazas que vulneren su salud 
mental y física. La actual crisis de los cuidados se encuentra sujeta a agravarse de acuerdo con el impacto económico que recaiga sobre las personas que lo ejercen. En ese sentido, es importante recordar que las políticas de cuidado deben ser evaluadas desde perspectivas transversales (Sojo, 2011).

Por lo que será necesario que las acciones sean coordinadas, más ahora que nunca, desde las políticas de empleo, transporte, vivienda, género, fiscal, educación y ocio, ya que el debilitamiento o descuido de un sector puede agudizar la crisis de cuidados al estar todas estas secciones mutuamente relacionadas.

Así mismo, es importante que los gobiernos dejen de invisibilizar los cuidados que son fundamentales en el ser de cualquier persona y, por lo tanto, se debe buscar que sea jerarquizado y remunerado, ya que es parte de lo que entendemos como economía.

En esa línea, se deben sumar esfuerzos para reconocer, distribuir y reducir los cuidados con ayuda del Estado y la sociedad civil. Es importante, además, que las políticas públicas que se implementen no solo sean de un lado hacia otro (arriba-abajo), sino que dialoguen y la voz de las personas que puedan ser afectadas sea consultada. Por último, frente al fin del bono demográfico y el inevitable proceso de envejecimiento de la región, es preciso implementar mejores políticas de salud pública para un envejecimiento sano y digno.

\section{Referencias}

Acosta, L. D. (2020). Capacidad de respuesta frente a la pandemia de COVID-19 en América Latina y el Caribe. Revista Panamericana de Salud Pública, (44), 1-8. https://doi.org/10.26633/ rpsp.2020.109

Álvarez, S. (2020). (In)movilidad en las Américas en tiempos de pandemia. Dossier: Reflexiones en torno al COVID-19. LASA Forum, 51(3), 17-23.

Aranco, N., Stampini, M., Ibarrarán, P., y Medellín, N. (2018). Panorama de envejecimiento y dependencia en América Latina y el Caribe. Resumen de Políticas. Banco Interamericano de Desarrollo. https://publications.iadb.org/publications/spanish/document/Panorama-de-envejecimiento-y-dependencia-en-America-Latina-y-el-Caribe.pdf 
Batthyany, K. (2020). Organización social del cuidado y crisis sanitaria en América Latina y el Caribe. Dossier: Reflexiones en torno al COVID-19. LASA Forum, 51(3), 24-27. https:// www.clacso.org/wp-content/uploads/2020/08/DossierCOVID19-4.pdf

Bettio, F., Simonazzi, A., \& Villa, P. (2006). Change in care regimes and female migration: the 'care drain'in the Mediterranean. Journal of European social policy, 16(3), 271-285. https://doi. org/10.1177/0958928706065598

Bohoslavsky, J. P. y Rulli, M. (2020). COVID-19, instituciones financieras internacionales y continuidad de las políticas androcéntricas en América Latina. Revista Estudios Feministas, 28(2). https://doi.org/10.1590/1806-9584-2020v28n273510

Buttler, J. (12 de abril de 2020). Rastros humanos en las superficies del mundo. Lobosuelto. http://lobosuelto.com/rastros-humanos-en-las-superficies-del-mundo-judith-butler/

Calderón, C. (2013). Redistribuir el cuidado: el desafío de las políticas. Cuadernos de la CEPAL, (101). http://hdl.handle. net/11362/27871

Camargo, L., \& Caicedo, H. (2012). “Informalidad: escape y exclusión” de Guillermo Perry et al. Sociedad y Economía, (23), 247-250.

Centeno, M. A. y Ferraro, A. E. (Eds.). (2013). State and nation making in Latin America and Spain: republics of the possible. Cambridge University Press.

Comisión Económica para América Latina y el Caribe, CEPAL. (2020a). Informe sobre el impacto económico en América Latina y el Caribe de la enfermedad por coronavirus (COVID-19). http:// hdl.handle.net/11362/45602

Comisión Económica para América Latina y el Caribe, CEPAL. (2020b). América Latina y el Caribe ante la pandemia del COVID-19: efectos económicos y sociales. Informe especial COVID-19, (1). http://hdl.handle.net/11362/45337

Comisión Económica para América Latina y el Caribe, CEPAL. (2020c). La pandemia del COVID-19 profundiza la crisis de los cuidados en América Latina y el Caribe. Informe. http://hdl. handle.net/11362/45335

Dean, H. (2008). Social policy and human rights: Re-thinking the engagement. Social Policy and Society, 7(1), 1-12. https://doi. org/10.1017/s147474640700396x 
Del Valle, A. H. (2010). Comparando regímenes de bienestar en América Latina. Revista Europea de Estudios Latinoamericanos y del Caribe/European Review of Latin American and Caribbean Studies, (88), 61-76. https://doi.org/10.18352/ erlacs.9596

Domínguez Alcón, C. (1998). Cuidado informal, redes de apoyo y políticas de vejez. Index de Enfermería, (23), 15-21. https:// core.ac.uk/download/pdf/16213324.pdf

Esping-Andersen, G. (1993). Las tres economías políticas del Estado de bienestar. Ediciones Alfons El Magnànim.

Esquivel, V. y Faur, E. (2014). Las lógicas del cuidado infantil. Entre las familias, el Estado y el mercado. ÍCONOS, 50, 211-224. https://www.redalyc.org/pdf/509/50931716015.pdf

Fleury, S. (2021). Estado sin ciudadanos. Seguridad social en América Latina. Ediciones Universidad Nacional de Lanús.

Filgueira, F. (1998). El nuevo modelo de prestaciones sociales en América Latina: eficiencia, residualismo y ciudadanía estratificada. Centroamérica en reestructuración. https://blogs-fcpolit. unr.edu.ar/politicasocial1/files/2015/03/Filgueira-El-nuevo-modelo-de-prestaciones-sociales-en-Am\%C3\%A9ricaLatina.pdf

Franzoni, J. M. (2005). Regímenes de bienestar en América Latina: consideraciones generales e itinerarios regionales. Revista Centro-Americano de Ciências Sociais, 4(2), 41-77. http://www. derechoshumanos.unlp.edu.ar/assets/files/documentos/ regimenes-de-bienestar-en-america-latina-consideraciones-generales-e-itinerarios-regionales.pdf

Franzoni, J. M. (2008). Welfare regimes in Latin America: Capturing constellations of markets, families, and policies. Latin American Politics and Society, 50(2), 67-100. https://doi. org/10.1111/j.1548-2456.2008.00013.x

Garay, C. (2016). Social policy expansion in Latin America. Cambridge University Press.

Gutiérrez, D., Martin, G., y Ñopo, H. (2020). El coronavirus y los retos para el trabajo de las mujeres en América Latina. Grupo de Análisis para el Desarrollo, GRADE. https://www.grade.org. pe/wp-content/uploads/GRADEdil10.pdf

Holland, A. C. y Schneider, B. R. (2017). Easy and hard redistribution: The political economy of welfare states in Latin Amer- 
ica. Perspectives on Politics, 15(4), 988-1006. https://doi. org/10.1017/s1537592717002122

Huber, E. (2009). Politics and inequality in Latin America. PS: Political Science and Politics, 42(4), 651-655. https://doi.org/10.1017/ s1049096509990084

Lamaute-Brisson, N. (2013). Redistribuir el cuidado: para un nexo de políticas públicas. En C. Calderón Magaña (Coord.), Redistribuir el cuidado: el desafío de las políticas (pp. 69-118). CEPAL

Lavell, A., Mansilla, E., Maskrey, A., y Ramírez, F. (2020). La construcción social de la pandemia COVID-19: desastre, acumulación de riesgos y políticas públicas. La Red (Red de Estudios Sociales en Prevención de Desastres en América Latina). https:// www.desenredando.org/covid19/Construcci\%C3\%B3n-social-pandemia-Covid19-desastre-riesgo-politicas-publicasRNI-LA-RED-23-04-2020.pdf

Mesa-Lago, C. (2008). History of social security in Latin America. En International Meeting on the History of Insurance Companies in the World (pp. 1-16). Fundación Mapfre.

Organización de Estados Americanos, OEA. (2020). Covid-19 en la vida de las mujeres: Emergencia global de los cuidados / Comisión Interamericana de Mujeres. http://www.oas.org/es/cim/ docs/Cuidados COVID19-ES.pdf

ONU-Mujeres y Comisión Económica para América Latina y el Caribe, CEPAL. (2020). Cuidados en América Latina y el Caribe en tiempos de COVID-19: hacia sistemas integrales para fortalecer la respuesta y la recuperación. http://hdl.handle. net/11362/45916

Perry, G., Maloney, W., Arias, O., Fajnzylber, P., Mason, A., y Saavedra-Chanduvi, J. (2007). Informalidad: escape y exclusión. Banco Mundial. https://documents1.worldbank.org/curated/en/889371468313790669/pdf/400080PUB0SPAN101O FFICIALOUSE0ONLY1.pdf

Pierson, C. (2005). 'Late industrializers' and the development of welfare regimes. Acta Politica, 40(4), 395-418. https://doi. org/10.1057/palgrave.ap.5500123

Pribble, J. (2011). Worlds apart: Social policy regimes in Latin America. Studies in Comparative International Development, 46(2), 191-216. https://doi.org/10.1007/s12116-010-9076-6 
Rossel, C. (2016). Desafíos demográficos para la organización social del cuidado y las políticas públicas. Serie Asuntos de Género, CEPAL. http://hdl.handle.net/11362/40239

Sojo, A. (2011). De la evanescencia a la mira: El cuidado como eje de políticas y de actores en América Latina. Serie Seminarios y conferencias, CEPAL. http://hdl.handle.net/11362/7023 